\title{
Fresh Perspectives on the Alcohol and HIV Nexus: A Call for Action in an Era of Increased Opportunities and Challenges
}

\author{
Charles D. H. Parry ${ }^{1,2}$ (1) Mark Tomlinson ${ }^{3} \cdot$ Kendall Bryant $^{4}$. \\ Mary Jane Rotherham-Borus ${ }^{5}$
}

Published online: 27 September 2017

(C) Springer Science+Business Media, LLC 2017

While there is now a much greater understanding of the links between alcohol and HIV than there was 10 years ago, there are still gaps in our understanding of how alcohol use and heavy drinking impacts on HIV and AIDS, both in terms of increasing the likelihood of acquiring HIV and also in progressing the disease among affected persons. Furthermore, we need to improve our knowledge of how best to intervene with persons who drink alcohol and who are not HIV positive, as well as among persons who are, and who are possibly on antiretroviral (ARV) medications. This may be of particular importance in settings with a limited range of medical resources to screen and retain individuals in care.

In 2008, a World Health Organization (WHO) technical consultation on alcohol and infectious diseases was held in Cape Town, South Africa. After reviewing the available evidence including specifically commissioned systematic reviews and meta-analyses it was concluded that heavy drinking patterns and/or alcohol use disorders worsened the disease course of HIV. However, while alcohol usage was associated consistently with the prevalence and incidence of

Charles D. H. Parry

cparry@mrc.ac.za

1 Alcohol Tobacco \& Other Drug Research Unit, South African Medical Research Council, Tygerberg, South Africa

2 Department of Psychiatry, Stellenbosch University, PO Box 19070, Tygerberg 7505, South Africa

3 Department of Psychology, Stellenbosch University, Stellenbosch, South Africa

4 HIV/AIDS and Alcohol Research Program, National Institute on Alcohol Abuse and Alcoholism, Bethesda, MD, USA

5 Global Center for Children and Families, Semel Institute and the Department of Psychiatry, University of California, Los Angeles (UCLA), Los Angeles, CA, USA
HIV, participants agreed that further research was needed to substantiate causality [1]. A year later, Rehm et al. [2] released the first burden of disease assessment that included a calculation of the effect of alcohol on HIV and AIDS. It estimated that that $1.4 \%$ of disability-adjusted life years (DALYS) lost due to alcohol in 2004 could be attributed to the effect of alcohol on the progression of HIV and AIDS. This was calculated using information on the effect of alcohol consumption, and particularly heavy drinking, on antiretroviral treatment adherence together with an estimation of the impact of failed adherence on survival [3].

In the years following the 2008 consultation and the highly-cited 2010 Addiction paper on alcohol and burden of disease [3], knowledge of risk relations between different dimensions of alcohol use and health outcomes used in global and national burden of disease estimates has increased substantially [4]. We are now in a better position to be able to conclude that there is a causal relationship between drinking and the acquisition of HIV, to quantify this relationship, and more accurately estimate the effect of drinking on HIV disease progression [4]. As a result, it is now expected that the 2017 WHO Global Status Report on Alcohol and Health will include an estimate of alcohol's impact on the acquisition of HIV and not just on HIV disease progression [4].

A second WHO consultation held in Cape Town in 2012, recognizing gaps in knowledge, identified three key priorities for research in the area of alcohol and HIV. First, it highlighted the need for prevalence studies among the HIV patient populations to obtain baseline information on patterns of drinking, sexual HIV risk behaviors and information on treatment, including adherence for those on antiretroviral therapy (ART). Second, it called for more randomized controlled trials (RCTs) to evaluate evidenced-based, alcoholfocused interventions on reducing the risk of acquiring HIV among uninfected persons. Third, it stressed the need for 
RCTs to evaluate evidenced-based alcohol-focused interventions on treatment adherence and response among persons initiating treatment for HIV [5]. A research guide was subsequently developed on how to improve HIV (and tuberculosis (TB)) prevention and treatment through alcohol-focused brief interventions [6]. In addition, training on the contents of the guide was provided under the auspices of the WHO in Moldova (2013), Namibia (2014) and Botswana (2016).

Kessler et al. [7] conducted multiple simulations using proven HIV and alcohol interventions and estimated the overall impact of changes in alcohol use on the potential health outcomes. The interventions included screening for hazardous alcohol consumption and offering a cognitive behavioral therapy (CBT) intervention to those screening positive. Such interventions could be targeted to HIV-infected patients at different points in their illness and treatment cascade (i.e., all HIV-infected persons attending a clinic, only those patients in the pre-ART stages of care, only those patients receiving ART, and only those with detectable viral loads). This could prevent many new infections, add thousands of quality-adjusted life years (QALYs), and yield an incrementally favorable cost-effectiveness ratio averting new infections [8]. Similar findings have emerged in randomized controlled trials with pregnant women in South Africa [9, 10]; Rotheram-Borus et al. [11]. Specifically, seropositive mothers reduce alcohol use and problematic drinking when visited by paraprofessional home visitors and increase their completion of tasks to Prevent Mother to Child Transmission (PMTCT) of HIV by 50\%. In addition, one of the most important findings is that there is significantly lower alcohol use and problematic drinking when their children are 5 years old. The intervention has also been shown to be cost-effective (Wynn et al. under review). These findings and others suggest that targeting effective treatment aimed at reducing hazardous alcohol consumption by subgroups of HIV-infected patients provides favorable value in comparison with other beneficial strategies for HIV prevention and control in many low resource regions with high rates of HIV and alcohol consumption.

Knowledge is also increasing regarding ways to better detect harmful use of alcohol in persons who are HIV positive and also regarding interventions that might be valuable in decreasing the impact of alcohol use, and especially heavy drinking, on HIV acquisition. These studies use a variety of intervention modalities (individual, group, and structural) which include cognitive-behavioral, motivational, electronic monitoring through phone applications (mHealth) among others at the individual level, and strategic interventions, such as stepped care in clinical settings using pharmacological agents. Multiple settings for community-based studies outside of medical contexts have been studied. These include bars, sexually transmitted infection (STI) clinics and other community settings with multiple methods for delivering interventions (e.g., telemedicine, ecological momentary assessment, electronic applications, and community-trained health professionals).

In terms of methodology, research conducted in Uganda has demonstrated the utility of using an alcohol biomarker, phosphatidylethanol (PEth), to augment self-report among HIV patients either on or not on ART [13]. Kalichman et al. [14] tested a three-hour alcohol and HIV risk reduction behavioral skills intervention for women and men who frequent informal drinking venues in South Africa. Positive outcomes at both 3- and 6- months were noted. Another study, also conducted in South Africa, among substance using patients attending an STI clinic compared a 20-minute HIV-STI educational session to a 60 -minute alcohol/HIV risk reduction counselling session. The study found significantly fewer incident STIs and significant reduction in vaginal and anal intercourse among patients who received the alcohol and sexual-HIV risk reduction counseling as compared to the control group. However, over time the differences between the two groups dissipated quicker for heavy alcohol drinkers as compared to lighter drinkers [15]. Finally, Wechsberg et al. [16] in another South African study found that women at risk of contracting HIV who had received a woman-focused ("Women's Health CoOp") intervention in the community were $32 \%$ more likely to be sober during their last sex act than women in the nutrition control group at 6-month follow up. However, Carrasco et al. [17] in a systematic review of the literature on HIV-alcohol risk reduction interventions in Africa point out that other studies found no significant intervention effects related to reduced alcohol use in sexual contexts and others had positive effects that quickly dissipated. Despite these conflicting findings, it is clear from Scott-Sheldon et al.'s [18] systematic review and meta-analysis, that among people living with HIV/AIDS (PLWH), alcohol use is significantly associated with unprotected sex. The conclusion we should reach is that this is an implementation issue and not an efficacy issue, and that there is a need for systematic follow-up studies and sustained interventions.

Other studies have focused on HIV patients in clinic settings who are either ART naïve or not and have been aimed at addressing hazardous drinking in order to impact on HIV disease progression, ART adherence, viral loads, treatment survival and other outcomes. A study of brief motivational intervention (MI) counseling in reducing alcohol consumption among persons living with HIV and AIDS in Uganda found that MI was effective only among women in reducing alcohol consumption [19]. The impact of this intervention on HIV specific outcomes such as medication adherence and viral load was not reported. There are several other major studies underway which hold potential in term guiding interventions designed to address hazardous drinking among HIV patients (primary outcome) and which also aim to report on 
secondary, HIV-related outcomes. The South African Medical Research Council in 2013 provided funding for a RCT to assess the efficacy of an alcohol-focused intervention for reducing alcohol consumption (primary outcome) and improving adherence to ART and HIV treatment outcomes (secondary outcomes) [20]. Primary outcomes are assessed via self-reported drinking as well as by measurement of PEth. Secondary outcomes are assessed via self-reported measures of adherence, biological markers of ART adherence (hair and blood) and biomarkers to assess viral load. Formative data from this project has indicated the utility of brief versions of the alcohol use disorders identification test [21] to identify excessive drinking among patients in HIV care in South Africa [22] and the acceptability of a brief alcohol-focused intervention for people living with HIV [23]. Further funding from the Welcome Trust has been obtained to take this work further in South Africa and Tanzania starting in September 2017, and in particular, to evaluate the operational feasibility, acceptability and preliminary impact of an alcohol and health system-focused intervention in these countries.

Another promising intervention study in HIV treatment settings currently underway is the "starting treatment for ethanol in primary care (STEP) trials" in the USA. For patients who are HIV positive with at-risk drinking or moderate alcohol use with liver disease, stepped care begins with a one session brief intervention accompanied by a telephone booster session 2 weeks later. Depending on pre-specified nonresponse criteria patients may be "stepped up" at the fourth week to receive four sessions of motivational enhancement therapy (MET) and may again be stepped up" at week 12 for addiction physician management (APM) and possibly also pharmacotherapy. Patients with an alcohol use disorder begin with APM. Non-responders may be "stepped up" to receive MET at week 4 and be "stepped up" again to receive higher level of care such as an intensive outpatient treatment program at week 12 [24]. This study uses self-reported measures of alcohol consumption, breathalyzer testing to measure current alcohol levels and PEth testing to determine chronic alcohol exposure. The secondary outcome is to change the Veterans Index Cohort Study (VACS) Index Score. This index is predictive of mortality, and combines age, traditional HIV and non-HIV biomarkers [25].

A systematic review published in 2016 of HIV-alcohol risk reduction interventions in sub-Saharan Africa, besides highlighting the need for further integrating HIV-alcohol risk reduction components into HIV prevention programming and to evaluate the efficacy of doing this, called for larger scale, multi-level interventions to reduce alcoholHIV risk [17]. A second review, also published in 2016 [26] investigated research conducted at alcohol venues to identify the structural and social factors associated with HIV risk in such venues and HIV prevention interventions.
They concluded that the venues themselves are important in influencing HIV risk behavior apart from the characteristics and behavior of individuals frequenting such venues, and that future research should look at developing and evaluating interventions targeting behavior change in drinking venues and other natural risk environments. According to Carrasco et al. [17] there is some evidence from other contexts that broader structural interventions, such as increasing alcohol prices/taxes, restricting marketing and decreasing availability to alcohol, can reduce alcohol consumption and lower rates of STIs and thus have the potential to reduce HIV acquisition. However, the effect of broader structural intervention such as these on alcohol-related HIV risk has been little studied. This may be because of the difficulties in separating out confounding factors, including the impact of co-occurring interventions, and the need for longitudinal research beyond the typical 3-5 years research funding cycles. The statistical challenges of teasing out the "noise" are also "not insignificant", but are not insurmountable.

The Office of AIDS Research at the NIH has focused on research in international settings in low-and middle-income countries in the past, as well as low resource settings found elsewhere. The current research priorities focus on preventing new infections, improving long-term treatment, developing potential cures, and addressing the complex comorbidities associated with HIV over the lifespan of individuals. The prevalence of alcohol use and its importance in increasing and sustaining the HIV epidemic is apparent and of increasing concern in the context of these research priorities. Even as medications for controlling the virus and achieving viral suppression are becoming more of a reality, misuse of alcohol still characterizes a large segment of the difficult-to-treat population. This increasing concern about addressing the complexity in treatment of co-factors and co-infections has been reflected in increased funding of current research on comorbidities. Of particular concern are other diseases such as hepatitis and TB as well as non-AIDS defining outcomes, such as increases in cardiovascular and hepatic mortality with other neurological complications. All of these diseases and their treatments and increased mortality are directly interactive and synergistic with unhealthy patterns of alcohol consumption among PLWH over their life-course.

As new ways of measuring both past and current alcohol exposure become available (e.g., transdermal biosensors and biomarkers), new and more effective interventions become possible. These include the timely delivery of advice on how individuals and key decision-makers may intervene to limit behavioral aspects of risk taking and reduce medication nonadherence as well as reduce the synergistic pathophysiology in the gut, liver, and brain and other organs. Cross-cutting research is needed to better understand how, and at what levels, alcohol influences comorbid conditions among people living with HIV in a variety of environments, and to 
understand the role of behavioral and biological alcoholrelated mechanisms in the context of common co-morbidities such at TB, hepatitis, depression, and other substance use. While it has been demonstrated that decreases in alcohol use improve HIV-relevant outcomes, new monitoring technologies through mHealth and telemedicine can sustain these changes and the uptake of appropriate medications and facilitate subsequent changes in healthy behaviors. These studies should also include assessment of the impact of the availability of state-of-the-art medications used to intervene with alcohol use disorders and treat common co-infections. These medications must be safe for both PLWH who take antiretroviral treatment and those individuals who continue to drink alcohol at hazardous or harmful levels.

In the future, it will be important to tailor interventions in the context of available resources. This will include capitalizing on advances in identifying and understanding subgroup variation to ensure that the risks of particularly vulnerable populations are considered when making decisions about the use of resources to implement effective interventions. This body of behavioral, biological, social, and cultural research should serve as a foundation for a next generation of multicomponent intervention studies to address alcohol use from transmission to treatment of HIV. Intervention studies should inform implementation efforts to improve provision of alcohol-related interventions and treatments for PLWH in healthcare settings and other community settings where hardto-reach populations such as high risk youth are found. By making further progress in understanding how alcohol use affects PLWH in the era of HIV as a chronic condition with a changing treatment landscape, this research should inform how we can mitigate transmission, achieve viral suppression, and avoid exacerbating common comorbidities of HIV and alcohol use. Ultimately, implementation research should facilitate progress toward achievable goals for sustained engagement of individuals in the widest range of effective care for alcohol misuse including those with alcohol use disorders and HIV.

This is a truly international special issue with papers reporting on data from a number of high-, middle- and low-income countries. Meeting the UNAIDS 90-90-90 targets is going to require increased efforts at reaching marginalized and difficult to reach groups. A number of papers in this special issue speak directly to this-a report on alcohol use and sexual risk behavior amongst migrant laborers in Kazakhstan (El Bassel et al. in this issue), HIV treatment adherence amongst injecting drug users in Vietnam ( $\mathrm{Li}$ et al. in this issue), and MSM Sex Workers in Dominican Republic (Tan et al. in this issue). This special issue also takes a broader view, with a systematic review on how people living with HIV/AIDS and using alcohol can be targeted with behavioral interventions (Scott-Sheldon et al. in this issue) as well as a priority setting exercise on research priorities for the intersection of alcohol and HIV/
AIDS (Gordon et al. in this issue). Finally, van Heerden and colleagues (in this issue) explore innovative new strategies to collect data and to deliver interventions.

Even if there is now greater knowledge regarding the link between alcohol and HIV and interventions that might be useful in reducing the impact of alcohol use on the acquisition of HIV and disease progression, there is no guarantee that this knowledge will influence policy and practice. What is also needed is more advocacy to ensure uptake of knowledge into action [33]. This could happen via publishing articles on these topics in publications with a broader readership like general nursing, medical and public health journals, but also through presentations (particularly keynote presentations, panel/discussion sessions and other oral presentations) at international conferences. While we have seen alcohol issues being presented at international AIDS conferences, they have often not been given much visibility. For example, at the $9^{\text {th }}$ International AIDS Society in Paris in July 2017 a review of the online scientific program revealed no oral presentations with "alcohol" in the title. One exception, where the topic of alcohol and HIV was given some prominence occurred at the 8th South African AIDS conference in July 2017. Here Dr Paul Shuper of the Centre for Addiction and Mental Health in Canada was given a slot in the 3rd plenary to speak on "Alcohol as the driver of the HIV epidemic in sub-Saharan Africa: Evidence and implications". Clearly, much more needs to be done to increase the visibility of the findings of research in the alcohol and HIV area and to ensure that there is sufficient debate about their implications for policy and practice.

Funding NIAAA Conference Grant (U13) Number U13AA023748 awarded to MJRB (UCLA) for a meeting on "Alcohol as a barrier to eliminating HIV" which was held prior to the International AIDS Conference in Durban, July 2016. It was at this conference that the idea of a special issue of a journal on alcohol and HIV was conceived. CDHP is supported by the South African Medical Research Council and the National Research Foundation, South Africa. MT is supported by the National Research Foundation, South Africa and is a Lead Investigator of the Centre of Excellence in Human Development, University Witwatersrand, South Africa.

\section{Compliance with Ethical Standards}

Conflicts of interest All authors declare that they have no conflicts of interest.

Ethical Approval This article does not contain any studies with human (or animal) participants undertaken by any of the authors.

\section{References}

1. Parry CDH, Rehm J, Poznyak V, Room R. Alcohol and infectious diseases: an overlooked causal linkage? Addiction. 2009;104:331-2. 
2. Rehm J, Anderson P, Kanteres F, Parry CD, Samokhvalov AV, Patra J. Alcohol, social development and infectious disease. Stockholm: Ministry of Health \& Social Affairs; 2009.

3. Rehm J, Baliunas D, Borges GLG, Graham K, Irving HM, Kehoe T, Parry CD, Patra J, Popova S, Poznyak V, Roerecke M, Room $\mathrm{R}$, Samokhvalov AV, Taylor B. The relation between different dimensions of alcohol consumption and burden of disease-an overview. Addiction. 2010;105:817-43.

4. Rehm J, Gmel GE, Gmel G, Hasan OSM, Imtiaz S, Popova S, Probst C, Roerecke M, Room R, Samokhvalov AV, Shield KD, Shuper PA. The relationship between different dimensions of alcohol use and the burden of disease-an update. Addiction. 2017;112:968-1001.

5. Parry CDH, Ferreira-Borges C, Poznyak V, Lönnroth K, Rehm J. The International Study on alcohol and infectious diseases: three priorities for research. Addiction. 2012;108:1-2.

6. Shuper PA, Rehm J. Improving HIV and TB prevention and treatment through alcohol-focused brief interventions. Toronto: Centre Addict Mental Health; 2013.

7. Kessler J, Ruggles K, Nucifora K, Li Roberts MS, et al. Targeting an alcohol intervention cost-effectively to persons living with HIV/ AIDS in East Africa. Alcohol Clin Exp Res. 2015;39:2179-88.

8. Kraemer KL. Can a behavioral alcohol intervention be delivered cost-effectively to persons living with HIV/AIDS in Sub-Saharan Africa? Alcohol Clin Exp Res. 2016;40:50-1.

9. Le Roux I, Tomlinson M, Mbewu N, Stewart J, Harwood J, Worthman CM, et al. Outcomes of home visits for pregnant township mothers and their infants in South Africa: a cluster randomised controlled trial. AIDS. 2013;27:1461-71.

10. Tomlinson M, Rotheram-Borus MJ, Le Roux IM, Youssef M, Nelson SH, Scheffler A, et al. 36 month outcomes of a generalist paraprofessional perinatal home visiting intervention in South Africa. Prev Sci. 2016;17:937-48.

11. Rotherham-Borus MJ., Tomlinson M, Le Roux I, Weichle T, Wynn A. Extending perinatal care for mothers, including mothers living with HIV (MLH), and their children through paraprofessional home visiting: RCT results over 5 years. Poster presented at the International AIDS Society Conference, Paris France, July 2017.

12. Wynn A, Rotheram-Borus MJ, Weichle T, Le Roux IM, Tomlinson M. Costs and benefits of a randomized controlled trial evaluating perinatal home visiting among South African Mothers/ Infants. Health Aff. Under review.

13. Hahn JA, Emenyonu NI, Fatch R, Muyindike WR, Kekiibina A, Carrico AW. Declining and rebounding unhealthy alcohol consumption during the first year of HIV care in rural Uganda, using phosphatidylethanol to augment self-report. Addiction. 2015;111:272-9.

14. Kalichman S, Simbayi L, Vermaak R, et al. Randomized trial of a community-based alcohol-related HIV risk-reduction intervention for men and women in Cape Town, South Africa. Ann Behav Med. 2008;36:270-9.

15. Kalichman S, Cain D, Eaton L, Jooste S, Simbayi LC. Randomized clinical trial of brief risk reduction counseling for sexually transmitted infection clinic patients in Cape Town, South Africa. Am J Public Health. 2011;101:e9-17.

16. Wechsberg WM, Jewkes R, Novak S, Kline T, Myers B, Browne FA, et al. A brief intervention for drug use, sexual risk behaviours, and violence prevention with vulnerable women in South Africa: a randomised trial of the women's health CoOp. BMJ Open. 2013;3:e02622.

17. Carrasco MA, Esser MB, Sparks A, Kaufman MR. HIV-alcohol risk reduction interventions in Sub-Saharan Africa: a systematic review of the literature and recommendations for a way forward. AIDS Behav. 2016;20:484-503.

18. Scott-Sheldon LA, Walstrom P, Carey KB, Johnson BT, Carey MP. The MASH Research Team. Alcohol use and sexual risk behaviors among individuals infected with HIV: a systematic review and meta-analysis 2012 to early 2013. Curr HIV/AIDS Rep. 2013;10:314-23.

19. Wandera B, Tumwesigye NB, Nankabirwa JI, Mafigiri DK, Parkes-Ratanshi RM, Kapiga S, et al. Efficacy of a single, brief alcohol reduction intervention among men and women living with HIV/AIDS and using alcohol in Kampala, Uganda: a randomized trial. J Int Assoc Provid AIDS Care. 2017;16:276-85.

20. Parry CDH, Morojele NK, Myers BJ, Kekwaletswe CT, Manda SOM, Sorsdahl K, Ramjee G, Hahn JA, Rehm J, Shuper PA. Efficacy of an alcohol-focused intervention for improving adherence to antiretroviral therapy (ART) and HIV treatment outcomes-a randomised controlled trial protocol. BMC Infect Dis. 2014;14:500.

21. Babor TF, Higgins-Biddle JC, Saunders JB, Monteiro MG. The alcohol use disorders identification test: guidelines for use in primary care. Geneva: World Health Organization, Department of Mental Health and Substance Abuse Dependence; 2001.

22. Morojele NK, Nkosi S, Kekwaletswe C, Shuper P, Manda SO, Myers B, Parry CDH. Utility of brief versions of the alcohol use disorders identification test (AUDIT) to identify excessive drinking among patients in HIV care in South Africa. J Stud Alcohol Drugs. 2017;78:88-96.

23. Myers B, Sorsdahl K, Morojele N, Kekwaletswe C, Shuper PA, Parry CDH. "In this thing I have everything I need": perceived acceptability of a brief alcohol-focused intervention for people living with HIV. AIDS Care. 2016;29:209-13.

24. Edelman EJ, Maisto SA, Hansen NB, Cutter CJ, Dziura J, Fiellin $\mathrm{LE}$, et al. The starting treatment for ethanol in primary care trials (STEP Trials): protocol for three parallel multi-site stepped care effectiveness studies for unhealthy alcohol use in HIV-positive patients. Contemp Clin Trials. 2017;52:80-90.

25. Marquine MJ, Umlauf A, Rooney A, Fazeli P, Gouax B, Woods SP, Letendre SL, et al. The veterans aging cohort study (VACS) index is associated with concurrent risk for neurocognitive impairment. JAIDS. 2014;65:190-7.

26. Pitpitan EV, Kalichman SC. Reducing HIV risks in the places where people drink: prevention interventions in alcohol venues. AIDS Behav. 2016;20:S119-33.

27. El Bassel N, Marotta P. (2017) Alcohol and sexual risk behaviors among male Central Asian labor migrants and non-migrants in Kazakhstan: implications for HIV Prevention. AIDS Behav. 2017; this issue.

28. Li L, Luo S, Lan C-W, Lin C, Le AT, Feng, N, Nguyen AT. Alcohol use, HIV treatment adherence, and sexual risk among people with a history of injecting drug use in Vietnam. AIDS Behav. 2017; this issue.

29. Tan D, Holloway IW, Gildner J, Jauregui J, Alvarez RG, Guilamo-Ramos V. Alcohol use; human immunodeficiency virus; social networks; sex work; Dominican Republic. AIDS Behav. 2017; this issue.

30. Scott-Sheldon LAJ, Carey KB, Johnson BT, Carey MP. Behavioral interventions targeting alcohol use among people living with HIV/AIDS: a systematic review and meta-analysis. AIDS Behav. 2017; this issue.

31. Gordon S, Rotherham-Borus MJ, Skeen S, Parry C, Bryant K, Tomlinson K. (2017) Research Priorities for the intersection of Alcohol and HIV/AIDS in Low and Middle Income Countries: A priority setting exercise. AIDS Behav. 2017; this issue.

32. Van Heerden A, Tomlinson M, Skeen S, Parry C, Bryant K, Rotherham Borus MJ. (2017) Innovation at the intersection of Alcohol and HIV research. AIDS Behav. 2017; this issue.

33. Parry CDH, Rehm J, Morojele NK. Is there a causal relationship between alcohol and HIV: implications for policy, practice and future research? Afr J Drug Alcohol Stud. 2010;9(2):81-91. 